\title{
Síndrome da morte súbita infantil em Pelotas de 2006 a 2013: uma análise descritiva
}

\author{
Sudden infant death syndrome in Pelotas from 2006 to 2013: a descriptive analysis
}

\author{
Rafaella de Lima Lorea ${ }^{1}$, Maurício Castro Pilger ${ }^{1}$, Milton Luiz Ceia ${ }^{2}$
}

Lorea RL, Pilger MC, Ceia ML. Síndrome da morte súbita infantil em Pelotas de 2006 a 2013: uma análise descritiva / Sudden infant death syndrome in Pelotas from 2006 to 2013: a descriptive analysis. Rev Med (São Paulo). 2017 jan.-mar.;96(1):27-31.

\begin{abstract}
RESUMO: A Síndrome da Morte Súbita Infantil (SMSI) ocupa a oitava posição entre as causas de anos potenciais de vida perdidos e as primeiras posições como causa de mortalidade infantil pósneonatal em países desenvolvidos ${ }^{1}$. O presente estudo objetiva conhecer as características socioepidemiológicas das crianças que foram a óbito por SMSI no município de Pelotas. Estudo observacional, retrospectivo, descritivo baseado nos dados da Secretaria Municipal de Saúde coletados através da aplicação de Fichas de Investigação de óbitos padronizadas pelo Ministério da Saúde de todos os casos de SMSI que ocorreram do ano de 2006 a 2013 em Pelotas, RS. Houve 37 óbitos registrados no período, o que representa um coeficiente de mortalidade por SMSI de 1,5 por mil. A média de idade materna foi de 23,5 anos $(\mathrm{dp}=5,2), 29(78 \%)$ eram fumantes e $23(62 \%)$ concederam aleitamento materno exclusivo até a data do óbito, 28 (76\%) tiverem seus bebês nascidos a termo. Dentre os 37 casos, 16 (43\%) vieram a falecer com menos de 1 mês de vida, 26 (70\%) dormiam junto aos pais e $23(61 \%)$ em decúbito lateral, enquanto que apenas $2(5 \%)$ em decúbito ventral e $16(43 \%)$ dos casos de SMSI ocorreram durante o inverno. O presente estudo é o único que abrange tamanha amostra ( 37 casos) de SMSI na cidade de Pelotas, a qual apresenta um coeficiente de mortalidade por essa patologia semelhante aos mais altos encontrados na literatura. Portanto, políticas públicas que visem a prevenção de SMSI em Pelotas são necessárias.
\end{abstract}

Descritores: Morte súbita do lactente; Idade gestacional; Decúbito dorsal, Aleitamento materno; Peso ao nascer.

\begin{abstract}
The Sudden Infant Death Syndrome (SIDS) ranks the eighth position as a cause of lost potential years of life and the first positions as a cause of post-neonatal infant mortality in developed countries. The present study aims to investigate the socio-epidemiological characteristics of children who died due to SIDS in the city of Pelotas. Observational, retrospective, descriptive study based on data from the Municipal Department of Health collected through the application of Deaths research Sheets according to standards from the Health Ministry of all cases of SIDS between 2006 and 2013 in Pelotas, RS. There were 37 registered deaths in the period, which represents a mortality coefficient by SIDS of 1.5 per thousand. The average of maternal age was 23,5 years ( $d p=5.2), 29(78 \%)$ were smokers and $23(62 \%)$ exclusively breastfed until the date of the death, $28(76 \%)$ had full-term babies. Amongst the 37 cases, 16 (43\%) died at less than one month old, $26(70 \%)$ were sleeping with their parents and $23(61 \%)$ in lateral decubitus, while only 2 $(5 \%)$ in prone position and $16(43 \%)$ of the SIDS cases occurred during the winter. The present study is the only with such sample (37 cases) of SIDS in the city of Pelotas, which presents a mortality coefficient for this pathology similar to the highest ones found in the literature. Thus, public policies which aim at SIDS in Pelotas are necessary.
\end{abstract}

Keywords: Sudden infant death; Gestational age; Supine position; Breast feeding; Birth weight.

Trabalho realizado na Universidade Federal de Pelotas. Apresentação oral e pôster no BRAICOMS-UNIFESP, out. 2016.

1. Acadêmicos de Medicina, Universidade Federal de Pelotas. E-mail: rafa_lorea@hotmail.com, mauriciopilger@gmail.com.

2. Médico, Mestre em Saúde Pública, Universidade Federal de Pelotas. Avenida Domingos de Almeida, 919 - Pelotas, RS. E-mail: mlceia@yahoo.com.br.

Endereço para correspondência: Rafaella de Lima Lorea. Rua Vereador Wolney Vieira, 211 - Pelotas, RS. E-mail: rafa_lorea@ hotmail.com 


\section{INTRODUÇÃO}

\begin{abstract}
Síndrome da Morte Súbita Infantil (SMSI) ocupa a oitava posição entre as causas de anos potenciais de vida perdidos e as primeiras posições como causa de mortalidade infantil pós-neonatal em países desenvolvidos ${ }^{1,2}$. No Brasil, há escassez de dados que nos permitam avaliar a influência de fatores socioculturais e geográficos na incidência dessa doença, pois os poucos estudos se concentram em estados do sul e sudeste ${ }^{3}$.

A SMSI foi definida por Beckwith ${ }^{4}$ como "a morte súbita inesperada de uma criança, na qual uma necropsia detalhada não consegue identificar uma causa adequada para o óbito". Esse evento passou a assumir papel relevante nos últimos anos a partir da queda dos índices de mortalidade infantil, devido a melhorias socioeconômicas, ambientais e na assistência médica, e à descoberta de que lactentes abaixo de 6 meses que dormem em posição de pronação possuem chance de $3 \mathrm{a}$ 9 vezes maior de sofrerem SMSI do que os que dormem em posição supina.
\end{abstract}

A definição de Beckwith ${ }^{4}$ torna praticamente impossível a realização de pesquisas epidemiológicas em nosso meio, haja vista a não realização de necropsias em crianças falecidas subitamente. Sendo assim, estudos brasileiros nessa área costumam adotar critério clínico para a classificação de SMSI, como "morte inesperada em criança assintomática ou com sintomas mínimos com menos de 24 horas de duração", o qual também foi usado para determinarmos a amostra nesse estudo ${ }^{3}$.

Nesse estudo, foram investigados todos os óbitos diagnosticados como SMSI na cidade de Pelotas entre os anos de 2006 e 2013, contabilizando 37 casos.

\section{MÉTODOS}

Estudou-se todos os casos de SMSI do ano de 2006 até o ano de 2013 no município de Pelotas-RS. Os dados foram coletados através da Secretaria Municipal de Saúde, que tem cobertura de $100 \%$ dos casos de óbito infantil através do Comitê de Investigação de Óbito Infantil, Fetal e Morte Materna (COMAI) desde 2003. O COMAI investiga os óbitos infantis através da aplicação das Fichas de Investigação de Óbitos (ficha de entrevista domiciliar, ficha de investigação hospitalar, ficha de investigação ambulatorial) padronizadas pelo Ministério da Saúde e colhendo informações da Declaração de Nascido Vivo, da Declaração de Óbito.

Foram excluídas do estudo crianças que não se enquadravam na definição clínica de SMSI. Ou seja, que apresentavam sintomas plausíveis de risco de vida por qualquer outra patologia.

Foram coletados dados referentes ao ano do óbito, idade materna, cor de pele, sexo, tabagismo materno, aleitamento materno exclusivo, posição em decúbito, idade da criança, peso ao nascimento, estação do ano em que ocorreu o óbito, idade gestacional e ocorrência de co-leito. A informação sobre peso ao nascer foi obtida através dos pais, dado obtido de maneira segura, conforme resultado de estudos prévios ${ }^{5}$ e confirmado pela Declaração de Nascido Vivo.

Após a coleta de dados, foi feita análise descritiva sobre a prevalência de SMSI no município de Pelotas.

\section{RESULTADOS}

Os 37 óbitos registrados ocorreram numa população estimada de 28.560 nascidos vivos, considerando média de 4.284 nascimentos por ano em famílias residentes em Pelotas, de acordo com a Secretaria Municipal de Saúde, o que representa um coeficiente de mortalidade por SMSI de 1,5 por mil.

A média de idade materna foi de 23,5 anos $(\mathrm{dp}=$ $5,2), 10,8 \%$ eram primigestas, 29 (78\%) eram fumantes e 23 (62\%) concederam aleitamento materno exclusivo até a data do óbito. 19 (51\%) mães eram da raça branca e $28(76 \%)$ tiverem seus bebês nascidos a termo. Dentre os 37 casos, 19 (51\%) crianças eram do sexo feminino, 17 (50\%) pesavam 2.906g em média (dp 619g), 16 (43\%) vieram a falecer com menos de um mês de vida, 26 (70\%) dormiam junto aos pais e 23 (61\%) em decúbito lateral, enquanto que apenas duas (5\%) em decúbito ventral. 16 (43\%) dos casos de SMSI ocorreram durante o inverno.

\section{DISCUSSÃO}

O presente estudo descritivo teve como objetivo principal conhecer características sócio-epidemiológicas, comportamentais e ambientais das crianças que foram a óbito por SMSI na cidade de Pelotas.

Deve-se levar em conta que alguns aspectos da metodologia utilizada podem subestimar ou superestimar o coeficiente de mortalidade, como a utilização de um critério clínico para definição de SMSI, haja vista que necropsias não foram utilizadas e nenhum exame microscópico foi realizado, pois, no Brasil, isto não consta como conduta obrigatória. Assim, o critério que classifica a causa como SMSI está à mercê dos médicos que acompanharam a criança e das informações obtidas através dos pais responsáveis. O coeficiente de SMSI de 
1,5 por mil não teve alteração diante de estudo longitudinal já realizado em Pelotas em $1982^{6}$ e assim como estudo realizado em Passo Fundo-RS ${ }^{7}$, apresenta um dos mais altos coeficientes internacionais. Por fim, para termos maior confiabilidade em dados de serviços estatísticos vitais, a melhoria na qualidade do preenchimento das fichas de óbito deve ser uma prioridade.

A maior prevalência de SMSI nos meses de inverno pode estar vinculada a possível etiologia da síndrome da SMSI infantil relacionada a doenças respiratórias virais, deficiências bioquímicas, hipotermia, as quais ocorrem mais comumente em meses mais frios ${ }^{3}$. Há estudos que indicam que esta prevalência nos meses de inverno esteja vinculada a quantidade de cobertas e agasalhos usados pelo lactente ${ }^{8}$.

Mães jovens e com mais de dois filhos são variáveis relacionadas à baixa renda e escolaridade, fatores que já foram comprovados pela literatura ${ }^{9,10}$ estarem associados a risco de ocorrência de SMSI.

Diversos estudos evidenciaram que o risco de SMSI é maior quando o lactente dorme na posição lateral do que na posição dorsal ${ }^{6,11}$ tendo em vista a maior probabilidade de rolar para a posição ventral do que para a posição dorsal ${ }^{12}$. Em 1991, foi lançada uma campanha na Inglaterra chamada "Back to sleep", que divulgava acerca da necessidade de crianças dormirem em decúbito dorsal em vista de evitar morte silenciosa por asfixia enquanto dormiam e outras possíveis complicações ${ }^{13}$. Através dessa política, o governo inglês conseguiu reduzir em $75 \%$ o número de óbitos por SMSI, resultados similares foram encontrados na Austrália e Nova Zelândia através da adesão da mesma campanha. Em nosso estudo, 23 (61\%) crianças assumiam a posição lateral para dormir, enquanto que $2(5 \%)$ dormiam em decúbito ventral - mostrando a preferência por dormir de lado, como apontado por demais estudos ${ }^{14}$. Em Pelotas, a campanha "back to sleep" foi realizada a partir do $2^{\circ}$ semestre de 2007 através da colocação de outdoors em vários pontos estratégicos da cidade, da promoção de palestras e exposição de cartazes junto às maternidades, hospitais e unidades básicas de saúde do município, além da distribuição de material de orientação aos pacientes. Atualmente, os outdoors foram retirados, mas a campanha é sustentada pelo material de divulgação e instrução médica para acadêmicos e demais profissionais da área da saúde. De acordo com a Secretaria Municipal de Saúde de Pelotas, do ano de 2005 ao ano de 2007, a média de casos de SMSI foi de 6,7 casos/ano, enquanto que do ano de 2008 a 2013, esse valor passou a ser 3,6 casos/ano. Em adição, em nenhum dos 20 casos ocorridos de 2005 a 2007, a mãe referiu decúbito dorsal do paciente ao dormir, enquanto que, dentre os 25 casos de 2008 a 2013, em 7 houve comprovação materna de que o paciente assumia a posição supina como hábito de sono. Estes valores podem refletir a uma mudança nos padrões de sono por repercussão da campanha. Porém, a amostra restrita ainda não é suficiente para concretizarmos o sucesso parcial da campanha, devendo ser realizados estudos mais específicos e melhor preenchimento das fichas de investigação de óbitos padronizadas pelo Ministério da Saúde para termos uma melhor perspectiva da campanha "back to sleep" em Pelotas. A necessidade desta política também é explícita por Rachel et al. ${ }^{15} \mathrm{e}$ Barsman et al. ${ }^{2}$, que indicam respectivamente falta de conhecimento e má orientação, perante os familiares, da influência da posição da crianças ao dormir. Isto se reflete através do resultado de que em $70 \%$ dos casos, as crianças dormiam junto aos pais, fator de risco para a ocorrência da SMSI também já comprovado ${ }^{7,11,16}$.

Shatet al. ${ }^{17}$ estimou que $20,7 \%$ dos casos de SMSI poderiam ser prevenidos se mães não fumassem durante gravidez. Estudos apontam que o tabagismo é um potente fator de risco para $\mathrm{SMSI}^{10,18}$, mas também um dos que mais facilmente pode ser evitado, fato que reitera importância de políticas públicas anti-tabagismo especialmente durante gestação. Em nosso estudo, encontramos que 29 (78\%) das mães eram tabagistas.

Identificamos que $16(43 \%)$ casos de SMSI ocorreram até o primeiro mês de vida da criança, fator concordante com alguns estudos ${ }^{19}$, mas discordante em relação a outros que apontam para nítida maior taxa de SMSI nos meses subsequentes ${ }^{20}$, o que requer maior investigação para que tenhamos uma melhor perspectiva acerca desta variável.

\section{CONCLUSÃO}

O presente estudo é o único que abrange tamanha amostra (37 casos) de SMSI na cidade de Pelotas. Deve-se enfatizar a campanha "back to sleep" na cidade de Pelotas, pois, apesar de não ter sido feito estudo para avaliar sua real repercussão, análises prévias apontam para uma melhora nos hábitos de sono a partir do ano em que ela foi adotada. A falta de estudos como este em demais cidades do Rio Grande do Sul limita análises comparativas e o estabelecimento de políticas de prevenção específicas para cada região, considerando suas características socioculturais, socioeconômicas e geográficas. Isso faz com que políticas intervencionistas a curto prazo sejam voltadas à orientação da posição em decúbito, do coleito, do aleitamento materno exclusivo e da cessação do tabagismo, enquanto que políticas de longo prazo visem à melhora das condições socioeconômicas da região. 
Lorea RL, et al. Síndrome da morte súbita infantil em Pelotas de 2006 a 2013.

Tabela 1. Descrição das variáveis estudadas

\begin{tabular}{|c|c|c|c|c|c|c|}
\hline Variável & $\mathbf{N}^{\circ}$ & $(\%)$ & Variável & & $\mathbf{N}^{\mathbf{0}}$ & $(\%)$ \\
\hline Idade materna em anos & & & Sexo & & & \\
\hline $16-19$ & 9 & 24 & & Masculino & 18 & 49 \\
\hline $20-23$ & 13 & 35 & & Feminino & 19 & 51 \\
\hline $24-27$ & 8 & 22 & Idade da criança & & & \\
\hline$>27$ & 7 & 19 & & $\leq 1 \mathrm{~m}$ & 16 & 43 \\
\hline Número de filhos & & & & $>1 \mathrm{~m} \mathrm{e} \leq 2 \mathrm{~m}$ & 11 & 30 \\
\hline 1 & 4 & 11 & & $>2 \mathrm{~m} \mathrm{e} \leq 3 \mathrm{~m}$ & 6 & 16 \\
\hline 2 & 13 & 35 & & $>3 \mathrm{~m} \mathrm{e} \leq 4 \mathrm{~m}$ & 1 & 3 \\
\hline 3 & 12 & 32 & & $>4 \mathrm{~m}$ & 3 & 8 \\
\hline 4 & 4 & 11 & Peso ao nasciment & & & \\
\hline$\geq 5$ & 4 & 11 & & $<2000 \mathrm{~g}$ & 3 & 8 \\
\hline & & & & $\geq 2000 \mathrm{~g}<2500 \mathrm{~g}$ & 7 & 19 \\
\hline Mãe fumante & & & & $\geq 2500 \mathrm{~g}<3000 \mathrm{~g}$ & 10 & 27 \\
\hline Sim & 29 & 78 & & $\geq 3000 \mathrm{~g}$ & 17 & 50 \\
\hline Não & 8 & 22 & Estação do ano & & & \\
\hline & & & & Outono & 10 & 27 \\
\hline & & & & Inverno & 16 & 43 \\
\hline Cor da pele & & & & Primavera & 7 & 19 \\
\hline Branca & 19 & 51 & & Verão & 4 & 11 \\
\hline Negra & 10 & 27 & Período do óbito & & & \\
\hline Parda & 8 & 22 & & Neonatal & 16 & 43 \\
\hline & & & & Pós-neonatal & 21 & 57 \\
\hline Aleitamento materno exclusivo até óbito & & & Idade gestacional & & & \\
\hline Sim & 23 & 62 & & $<32^{\mathrm{a}}$ & 2 & 6 \\
\hline Não & 14 & 38 & & $>32^{\mathrm{a}} \mathrm{e} \leq 36^{\mathrm{a}}$ & 7 & 19 \\
\hline & & & & $>37^{\mathrm{a}} \mathrm{e} \leq 41^{\mathrm{a}}$ & 28 & 76 \\
\hline Posição em decúbito & & & Co Leito & & & \\
\hline Lateral & 23 & 61 & & Sim & 26 & 70 \\
\hline Dorsal & 7 & 19 & & Não & 7 & 19 \\
\hline Ventral & 2 & 5 & & Ignorado & 4 & 11 \\
\hline Ignorado & 5 & 5 & & & & \\
\hline
\end{tabular}

\section{REFERÊNCIAS}

1. McVea KL, Turner PD, Peppler DK. The role of breastfeeding in sudden infant death syndrome. J Hum Lact. 2000;16:13-20. doi: 10.1177/089033440001600104.

2. Barsman SG, Dowling DA, Damato EG, Czeck P. Neonatal Nurses' Beliefs, Knowledge, and Practices in Relation to Sudden
Infant Death Syndrome Risk-Reduction Recommendations. Adv Neonatal Care. 2015;15(3):209-19. doi: 10.1097/ ANC.0000000000000160.

3. Victora CG, Nobre LC, Lombardi C, Teixeira AM, Fuchs SMC, Moreira LB, et al. Quadro epidemiológico das mortes 
súbitas na infância em cidades gaúchas (Brasil). Rev Saúde Pública. 1987;21:490-6. http://hdl.handle.net/10183/71389.

4. Beckwith JB. Observations on the pathological anatomy of the sudden infant death syndrome. Seattle: University of Washington Press; 1970.

5. Victora CG, Barros FC. As mães lembram o peso ao nascer de seus filhos? Rev Saúde Pública. 1985;19(3):195-200. http://dx.doi.org/10.1590/S0034-89101985000300001.

6. Victora CG, Barros FC, Martines JC. Estudo longitudinal das crianças nascidas em 1982 em Pelotas, RS, Brasil: metodologia e resultados preliminares. Rev Saúde Pública. 1985;19(1):5868. http://dx.doi.org/10.1590/S0034-89101985000100007.

7. Geib LTC, Nunes ML. Incidência da síndrome da morte súbita em coorte de lactentes. J Pediatr (Rio J.). 2006,82(1):21-6. http://dx.doi.org/10.2223/JPED.1432.

8. Gutiérrez CA. Síndrome de la muerte súbita del lactante. Medicina de Família.net. 2001 [citado 10 fev. 2015]. Disponível em: http://www.zonadesalud.org.

9. Spencer S, Logan S. Sudden unexpected death in infancy and socioeconomic status: a systematic review. J Epidemiol Community Health. 2004;58(5):366-73. http://dx.doi. org/10.1136/jech.2003.011551.

10. Rocca RM, Bosch FJ, Henson DC, Reyes HP, Conde $\mathrm{AM}$, Risso RM, et al. Adherence to recommendations to reduce the risk of sudden infant death syndrome. Rev Chil Pediatr. 2014;85(4):462-9. doi: 10.4067/S037041062014000400009.

11. Horne RS, Hauck FR, Moon RY. Sudden infant death syndrome and advice for safe sleeping. BMJ. 2015;350:h1989. doi: https://doi.org/10.1136/bmj.h1989.

12. Scragg RK, Mitchell EA. Side sleeping position and bed sharing in the sudden infant death syndrome. Ann Med. 1998;30(4):345-9.

13. Jones WM. Supine and prone infant positioning: a winning combination. J Perinat Educ. 2004;13(1):10-20. doi:

\subsection{4/105812404X109357.}

14. Geib CTL, Nunes LM. Hábitos de sono relacionados à síndrome da morte súbita do lactente: estudo populacional. Cad Saúde Pública. 2006;22(2):415-23. http://dx.doi. org/10.1590/S0102-311X2006000200019.

15. Moon, YR, Oden, PR, Joyner, LB. Qualitative analysis of beliefs and perspections about sudden infant death syndrome in african-american mothers: Implications for safe sleep recommendations. J Pedriatr. 2010;157:92-7. doi: 10.1016/j. jpeds.2010.01.027.

16. Möllborg P, Wennergren G, Almqvist P, Alm B. Bed sharing is more common in sudden infant death syndrome than in explained sudden unexpected deaths in infancy. Acta Paediatr. 2015;104(8):777-83. 10. doi: 10.1111/apa.13021.

17. Shah, T, Sullivan K, Carter, J. Sudden infant death syndrome and reportes maternal smoking during pregnancy. Am J Public Health. 2006;96(10):1757-9. doi: 10.2105/ AJPH.2005.073213.

18. Cerpa VJ, Aylwin ML, Beltrán-Castillo S, Bravo EU, Llona IR, Richerson GB, et al. Prenatal-perinatal nicotine alters neonatal raphe neurons: meaning for sudden infant death syndrome. Am J Respir Cell Mol Biol. 2015;53(4):489-99.. doi: 10.1165/rcmb.2014-0329OC.

19. Rodríguez-Alarcón J, Melchor JC, Linares A, Aranguren G, Quintanilla M, Fernández-Llebrez L, de laGrindara A, Rodríguez-Soriano J. Early neonatal sudden death or near death syndrome. An epidemiological study of 29 cases. Acta Paediatr. 1994;83:704-8. doi: 10.1111/j.1651-2227.1994. tb13123.x.

20. American Academy of Pediatrics. Task force on infant sleep position and sudden infant death syndrome. Changing concepts of sudden infant death syndrome: implications for infant sleeping environment and sleep position. Pediatrics. 2000;105:650-6. Available from: http://pediatrics. aappublications.org/content/pediatrics/105/3/650.full.pdf 